Proceedings

\title{
Capacitive Contact Sensor on an Elastic Polymer Sheet ${ }^{+}$
}

\author{
Herbert Enser *, Christina Offenzeller, Marcel Knoll, Wolfgang Hilber and Bernhard Jakoby \\ Institute for Microelectronics and Microsensors, Johannes Kepler University Linz, 4020 Linz, Austria; \\ Christina.offenzeller@jku.at (C.O.); marcel.knoll@jku.at (M.K.); wolfgang.hilber@jku.at (W.H.); \\ bernhard.jakoby@jku.at (B.J.) \\ * Correspondence: herbert.enser@jku.at; Tel.: +43-732-2468-6262 \\ + Presented at the Eurosensors 2018 Conference, Graz, Austria, 9-12 September 2018. \\ Published: 13 December 2018
}

\begin{abstract}
There is an increasing need for embedded sensors integrated into parts and mechanical components, which are often polymer based. We investigated a solution to print capacitive sensors onto elastic polymer sheets to monitor the physical contact with other adjacent components. The capacitive sensors are oriented in an array across the surface of said sheet to monitor the contact and the distance to a neighboring electrically conductive object. In the particularly investigated setup, the sheet is embedded between to metallic (aluminum) plates and the capacitance of the sensor is changed due to the compression of the dielectric material between the interdigital fingers constituting the sensor.
\end{abstract}

Keywords: printed sensors; embedded sensors; printed electronics; printed interdigital sensor; printed capacitive sensor; printed pressure sensor

\section{Introduction}

We investigated a design of a printed capacitive interdigital contact and pressure sensor integrated into an elastic polymer sheet. In recent years, remarkable progress has been made in the field of printed electronics mostly on flexible substrates. A large number of low temperature curable conductive inks and pastes is already available. Low temperatures here refers to temperatures below $200{ }^{\circ} \mathrm{C}$ curing temperature. These inks are formulated as polymer matrices with conductive filler particles. This particles are mostly in the $\mu \mathrm{m}$ to $\mathrm{nm}$ range and are either spherical or flakes made of silver [1], gold [2], nickel [3], copper [4] particle free reactive silver [5] silver nano wire [6] conductive polymers [7-9] and carbon [10,11] based materials. Many electronic structures and sensors, which required cost intensive semiconductor fabrication, can now be printed, partially or fully, for example OLEDs [12], electrochromic displays [13], RFID tags [14], and polymer solar cells [15]. Most of these structures are printed on foils and in particular polymer sheets as considered in this work. The most problematic side effect are spurious capacitances, since the measured capacitance results from a network of various partial capacitances each being potentially sensitive to pressure and deformation.

\section{Materials and Methods}

The capacitive sensors in the form of interdigital finger structures investigated in this paper are made of silver polymer pastes and are fabricated via screen-printing. The elastic polymer substrate on top of which the sensor is printed in the present investigation is made from a stack from various materials, but the top most material is PET (polyethylene terephthalate). This ensures that a rather unproblematic printing process can be used. The ink used to print the capacitive sensors is a formulation provided by Henkel, PF050, and is optimized for printing onto polymers. Manufacturing 
of the sensor is realized by means of screen-printing with the semi-automatic screen print machine RokuPrint SD-05, where the ink has to be cured in a standard convection oven at $120^{\circ} \mathrm{C}$ for $10 \mathrm{~min}$. Since the substrate was chosen to be an elastic polymer compound with various thermal expansion coefficients, a jig was used to hold the sample in place while in the oven.

Figure 1 depicts three capacitive interdigital finger structures with different impedances, due to the difference in connection path length. The sheet thickness of the printed structure is $4 \mu \mathrm{m}$, and is covered with a $20 \mu \mathrm{m}$ transparent protection layer, which was chosen to be either a standard adhesive foil or, alternatively, was realized as a polymer coating on polyethylene basis using blade coating.

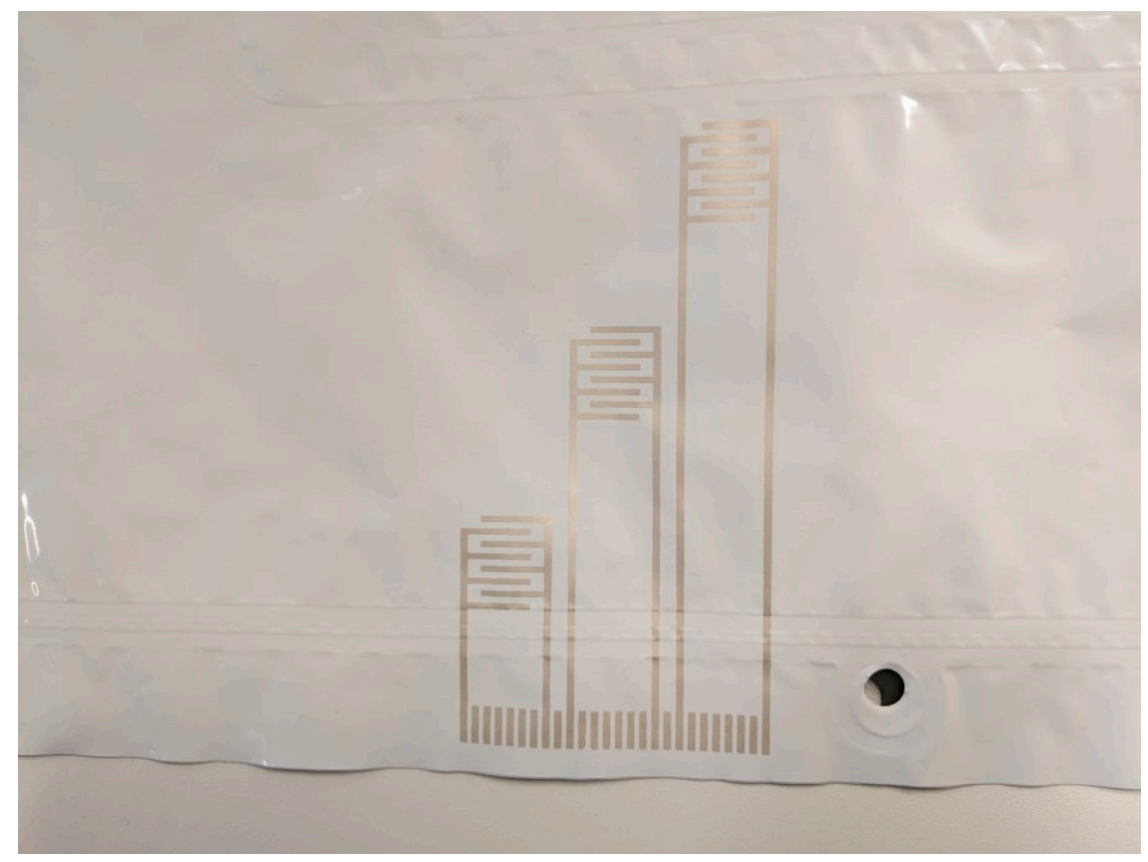

Figure 1. Sample of a printed capacitive interdigital pressure and contact sensor on an elastic polymer sheet. Sheet thickness of the printed sensors is $4 \mu \mathrm{m}$, and the material of choice is a polymer matrix paste with silver particles (Henkel PF-050). The capacitive sensor is covered with a transparent protection layer with a thickness of $20 \mu \mathrm{m}$. This protection layer is crucial for the function of the sensor, as well as for protection.

\section{Results}

Figure 2 shows a measurement of the absolute capacitance change on the three interdigital spots across the sample. For this measurement, the sheet was placed between two aluminum plates which were pressed together by means of loading weights, where a maximum mass of $5 \mathrm{~kg}$ was placed on a total plate area of $170 \mathrm{~cm}^{2}$ (corresponding to a maximum pressure of $\sim 2900 \mathrm{~Pa}$ ). The right most sample depicted in Figure 1 has a higher sensitivity than the other two, where their sensitivity decreases with decreasing contact path length. This contact path length results in a difference in absolute capacitance, and thus results in a change in sensitivity. All three sensors, however, show that a change in contact pressure from the elastic sheet to the aluminum enclosure results in a change of the measured capacitance. 


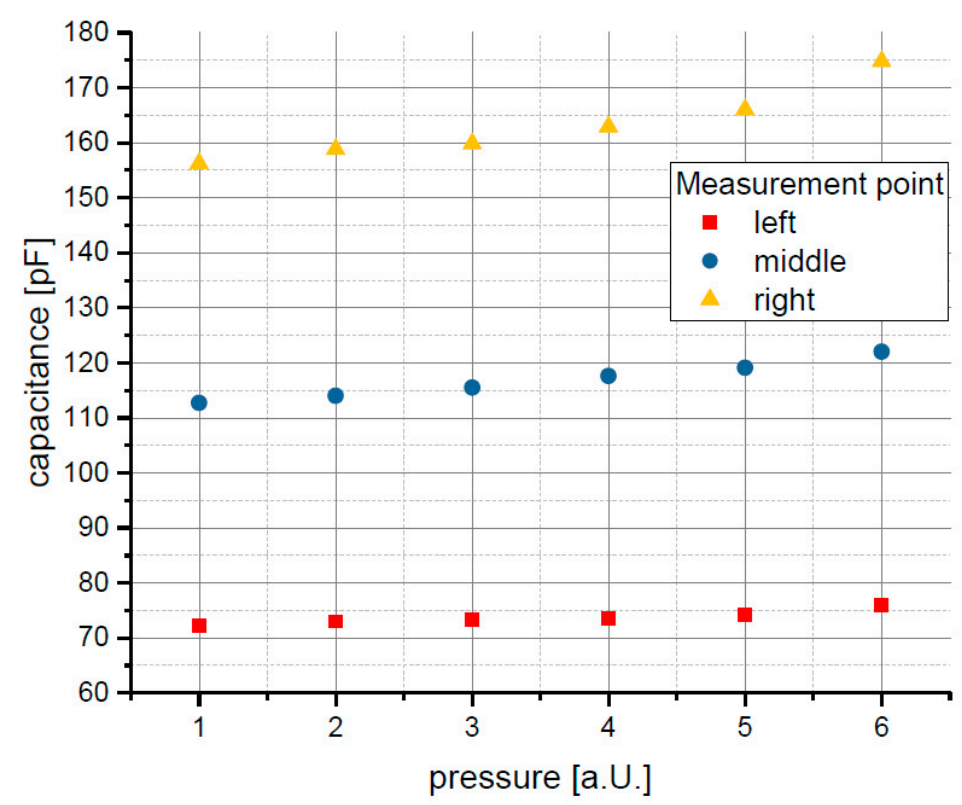

Figure 2. Response of the three sensors, shown in Figure 1. One can see that with increase in external pressure, all of the sensors show an appropriate response. However, the difference in absolute results in a difference in sensor sensitivity, where the right most sensor with the longest connection path has the highest sensitivity.

\section{Discussion and Conclusions}

The measurement of the interdigital capacitive sensor shows that an increasing contact pressure yields an increase of the capacitance. This is supposedly due to the squeezing effect on the covering foil on top of the electrodes. The investigation showed that this simple technology, which is considered to be used to realize general conductive patterns on polymer sheets, can also be utilized to monitor contact or, more general, pressure regarding adjacent conductive parts. The measurement of contact as well as pressure can be performed with just a one-layer sensor solution coupled with a flexible covering dielectric, in this case a transparent foil, but also a spray coating for example, is possible and viable.

Author Contributions: All authors jointly conceived and designed the experiments, where H.E. performed the experiments and analyzed the data; M.K and C.O. contributed reagents/materials/analysis tools; H.E. and B.J. wrote the paper.

Acknowledgments: This work has been supported by the Linz Center of Mechatronics (LCM) in the framework of the Austrian COMET-K2 programme and the Austrian. Research Promotion Agency (FFG-project No. 5834690).

Conflicts of Interest: The authors declare no conflict of interest.

\section{References}

1. Magdassi, S.; Grouchko, M.; Berezin, O.; Kamyshny, A. Triggering the sintering of silver nanoparticles at room temperature. ACS Nano 2010, 4, 1943-1948.

2 Huang, D.; Liao, F.; Molesa, S.; Redinger, D.; Subramanian, V. Plastic-compatible low resistance printable gold nanoparticle conductors for flexible electronics. J. Electrochem. Soc. 2003, 150, G412-G417.

3. Park, S.H.; Kim, H.S. Flash light sintering of nickel nanoparticles for printed electronics. Thin Solid Films 2014, 550, 575-581.

4 Ryu, J.; Kim, H.S.; Hahn, H.T. Reactive sintering of copper nanoparticles using intense pulsed light for printed electronics. J. Electron. Mater. 2011. 40, 42-50 
5. Lessing, J.; Glavan, A.C.; Walker, S.B.; Keplinger, C.; Lewis, J.A.; Whitesides, G.M. Inkjet Printing of conductive inks with high lateral resolution on omniphobic "Rf paper" for paper-based electronics and MEMS. Adv. Mater. 2014, 26, 4677-4682.

6. Finn, D.J.; Lotya, M.; Coleman, J.N. Inkjet printing of silver nanowire networks. ACS Appl. Mater. Interfaces 2015, 7, 9254-9261.

7. Vitoratos, E.; Sakkopoulos, S.; Paliatsas, N.; Emmanouil, K.; Choulis, S.A. Conductivity degradation study of PEDOT: PSS films under heat treatment in helium and atmospheric air. Open J. Org. Polym. Mater. 2012, 2, 7-11.

8. Yin, H.-E.; Huang, F.-H.; Chiu, W.-Y. Hydrophobic and flexible conductive films consisting of PEDOT: PSSPBA/fluorine-modified silica and their performance in weather stability. J. Mater. Chem. 2012, 22, 14042.

9. Reyes-Reyes, M.; Cruz-Cruz, I.; López-Sandoval, R. Enhancement of the electrical conductivity in PEDOT: PSS films by the addition of dimethyl sulfate. J. Phys. Chem. C 2010, 114, 20220-20224.

10. Feng, J.; Chan, C.-M. Double positive temperature coefficient effects of carbon black-filled polymer blends containing two semicrystalline polymers. Polymer 2000, 41, 4559-4565.

11. Enser, H.; Kulha, P.; Sell, J.K.; Schatzl-Linder, M.; Strauß, B.; Hilber, W.; Jakoby, B. Printed strain gauges embedded in organic coatings-Analysis of gauge factor and temperature dependence. Sens. Actuators A Phys. 2018, 276, 137-143.

12 Kopola, P.; Aernouts, T.; Sliz, R.; Guillerez, S.; Ylikunnari, M.; Cheyns, D.; Välimäki, M.; Tuomikoski, M.; Hast, J.; Jabbour, G.; et al. Gravure printed flexible organic photovoltaic modules. Sol. Energy Mater. Sol. Cells 2011, 95, 1344-1347.

13. Kawahara, J.; Ersman, P.A.; Engquist, I.; Berggren, M. Improving the color switch contrast in PEDOT: PSSbased electrochromic displays. Org. Electron. 2012, 13, 469-474.

14 Molesa, S.; Redinger, D.R.; Huang, D.C.; Subramanian, V. High-quality inkjet-printed multilevel interconnects and inductive components on plastic for ultra-low-cost RFID applications. MRS Online Proc. Libr. Arch. 2003, 769, H8.3.

15. Krebs, F.C.; Jørgensen, M.; Norrman, K.; Hagemann, O.; Alstrup, J.; Nielsen, T.D.; Fyenbo, J.; Larsen, K.; Kristensen, J. A complete process for production of flexible large area polymer solar cells entirely using screen printing -First public demonstration. Sol. Energy Mater. Sol. Cells 2009, 93, 422-441.

(C) 2018 by the authors. Licensee MDPI, Basel, Switzerland. This article is an open access article distributed under the terms and conditions of the Creative Commons Attribution (CC BY) license (http://creativecommons.org/licenses/by/4.0/). 